Check for updates

Cite this: RSC Adv., 2018, 8, 16314

\title{
Assessment of EtQxBox complexation in solution by steady-state and time-resolved fluorescence spectroscopy $\dagger$
}

\author{
Arianna Aprile, $\hbar^{a}$ Giovanna Palermo, (ID $\ddagger^{a}$ Antonio De Luca, (ID ${ }^{\text {ab }}$ Roberta Pinalli, (D) \\ Enrico Dalcanale (D) ${ }^{c}$ and Pasquale Pagliusi (D) *ab
}

\begin{abstract}
Reliable chemical sensors with high selectivity and sensitivity toward specific target molecules require rational synthesis of receptors, in-depth characterization of their complexation abilities and highly efficient transduction of the molecular recognition event. Here we report a steady-state and timeresolved fluorescence investigation of EtQxBox, a fluorescent conformationally blocked quinoxalinebased cavitand, aimed at assessing its selectivity toward aromatic versus non-aromatic analytes in solution. Fluorescence quenching of the EtQxBox in acetone is observed at increasing concentration of both aromatic (i.e. benzonitrile) and aliphatic (i.e. acetonitrile) compounds. The combination with fluorescence lifetime measurements permits to discriminate the predominantly static quenching of the aromatic analyte, due to non-fluorescent host-guest complex formation, from the mostly dynamic quenching of the non-aromatic compound, resulting from aspecific diffusive collisions between the fluorophore and the quencher. The equilibrium association constants for both the complexes have been estimated using Stern-Volmer model.
\end{abstract}

Received 3rd April 2018

Accepted 25th April 2018

DOI: $10.1039 / \mathrm{c} 8 \mathrm{ra0} 2875 \mathrm{c}$

rsc.li/rsc-advances

\section{Introduction}

Chemical sensing is of foremost importance for global health, because of its relevance in environmental monitoring, security and healthcare, ${ }^{1}$ yet is a major challenge for its strict requirements in terms of selectivity, sensitivity and ruggedness. ${ }^{2}$ In this field the exploitation of molecular receptors as sensing materials is attractive to address the selectivity issue. The conventional lock-and-key approach for selective chemical sensors design is based on the synthesis of a specific receptor wherein the analyte of interest is bound selectively. ${ }^{3-5}$ To create this specificity it is necessary to exploit molecular recognition between species that complement each other in size, shape and chemical functionality, which is further transduced with high fidelity and sensitivity into a physical signal. ${ }^{6-8}$ Among the different transduction modes, those based on fluorescence present many advantages: they are very sensitive (even single molecule detection is possible, under special conditions), low cost, easily performed, versatile, offering sub-nanometer spatial

\footnotetext{
${ }^{a}$ Department of Physics, University of Calabria, Ponte P. Bucci, Cubo 33B, 87036, Rende, CS, Italy.E-mail: pasquale.pagliusi@fis.unical.it

${ }^{b}$ CNR Nanotec - Institute of Nanotechnology, U.O.S. Cosenza, 87036 Rende, CS, Italy

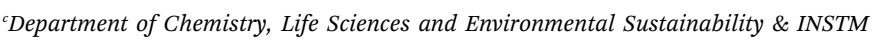
RU Parma, Parco delle Scienze 17/A, 43124 Parma, Italy

$\dagger$ Electronic supplementary information (ESI) available. See DOI: 10.1039/c8ra02875c

$\$$ A. Aprile and G. Palermo contributed equally to this work.
}

resolution with submicron visualization and sub-millisecond temporal resolution. ${ }^{9-12}$ Often, the chemical sensors work in the sense of dye displacement upon recognition event, therefore the receptor is required for selective binding of the substrate, while the fluorophore provides the means of signaling this binding, whether by fluorescent enhancement or inhibition..$^{13,14}$ For this purpose several fluorescent receptors have been synthesized and their complexation capabilities have been probed and demonstrated by fluorescence spectroscopy. ${ }^{10,11}$ Among them, a novel conformationally vase-blocked quinoxaline cavitand, namely EtQxBox, (Chart 1$)^{15}$ has demonstrated strong chemically affinity with aromatic volatile organic compounds (VOCs) such as benzene and its derivatives, namely BTEX (benzene, toluene, ethylbenzene and xylenes). ${ }^{15-17}$

Its rigidified cavity of molecular dimensions favors the intercalation of a specific class of complementary ligands due to a synergy of different weak interactions, like $\mathrm{C}-\mathrm{H} \cdots \pi$ and $\mathrm{C}-$ $\mathrm{H} \cdots \mathrm{N} \cdot{ }^{15}$ Combining the EtQxBox selectivity and sensitivity with its intrinsic fluorescence activity offers advantages in terms of host-guest binding transduction.

Herein, we report a comparative fluorescence spectroscopy investigation of an undecyl-footed EtQxBox (Chart 1) exposed to aromatic and aliphatic analytes, namely benzonitrile and acetonitrile, in acetone solution (for preparation and characterization see ESI†). Steady-state emission spectra show that both analytes yield quenching of the EtQxBox fluorescence. Indeed, beside the ground-state complex formation (static quenching), fluorescence quenching could also result from 


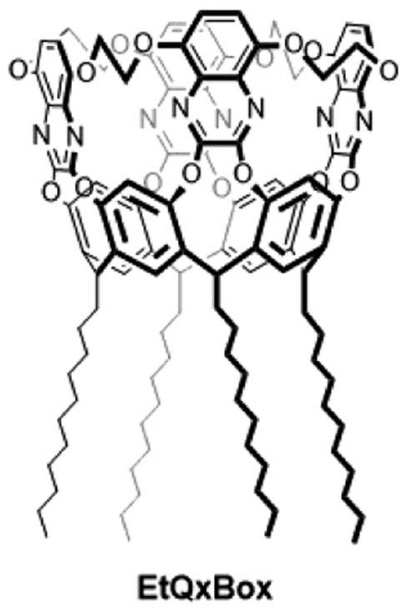

Chart 1 Structure of undecyl-footed EtQxBox cavitand, in which ethylendioxy bridges are introduced between the four quinoxaline wings to reduce the conformational breathing of the cavity.

collisional fluorophore-quencher encounters during the lifetime of the excited state (dynamic or collisional quenching). Time-resolved fluorescence spectroscopy allows to distinguish between static and dynamic quenching processes, since only the latter affects the fluorescence lifetime, and, in combination with the static measurements, provides quantitative assessment of both the collisional quenching constant and the association constant for complex formation. The analyses confirm that EtQxBox is quenched both by collisions and by complex formation with the analytes. Nevertheless, while dynamic quenching rates for the two analytes are equal, the association constants are ten times larger and three times smaller, for benzonitrile and acetonitrile, respectively.

\section{Experimental}

\section{Materials and chemicals}

The EtQxBox used in this work was synthesized using a literature procedure. ${ }^{15}$ In this case, the starting resorcinarene was functionalized at lower rim with four undecyl chains instead of four hexyl chains. EtQxBox was purified through column chromatography ( $\mathrm{SiO}_{2}$, acetone), and obtained as a yellow solid in $13 \%$ yield. Acetone, benzonitrile and acetonitrile, all of analytical reagent grade, were supplied by Sigma-Aldrich and used as received without further purification.

\section{Instrumentation}

The fluorescence measurements were carried out at $298 \mathrm{~K}$ on sample solutions placed in $10 \mathrm{~mm}$-thick quartz cuvette. A frequency-tripled $\left(\lambda_{\text {exc }}=355 \mathrm{~nm}\right.$, beam waist $\left.=4 \mathrm{~mm}\right) \mathrm{Q}$-switch Nd:YAG laser (Brio, Quantel) with $4 \mathrm{~ns}$ pulse duration and $20 \mathrm{~Hz}$ repetition rate was used to investigate the steady-state fluorescence, whose spectra were recorded with a fiber-spectrometer (USB200+UV-Vis, Ocean Optics, 600 lines per mm, $25 \mu \mathrm{m}$ slit) in the range $320-750 \mathrm{~nm}$. Time-resolved fluorescence spectroscopy measurements have been carried out by irradiating the samples with 100 fs laser pulses (Coherent Libra + OPerA Solo, $\lambda_{\text {exc }}=355 \mathrm{~nm}$, beam waist $=4 \mathrm{~mm}, P=20 \mu \mathrm{J}$, rep. rate $=1 \mathrm{k} \mathrm{Hz}$ ) and collecting the emission with a spectrograph (Acton SP2358i, $0.3 \mathrm{~m}$ focal length, 150 lines per $\mathrm{mm}, 20 \mu \mathrm{m}$ slit) equipped with a streak camera (Hamamatsu, C10910-01 + Slow Single Sweep module M10913-01 + CCD ORCA-R2) in the spectral range 470$570 \mathrm{~nm}$ and temporal window of $100 \mathrm{~ns}$.

\section{Results and discussion}

The steady-state fluorescence spectra of the EtQxBox solution $(0.5 \mathrm{mM})$ in acetone are reported for different concentrations of benzonitrile $\left(\mathrm{C}_{6} \mathrm{H}_{5} \mathrm{CN}\right.$, Fig. 1a) and acetonitrile $\left(\mathrm{CH}_{3} \mathrm{CN}\right.$, Fig. 1b). The emission band of EtQxBox in acetone, between 475 and $600 \mathrm{~nm}$, does not exhibit spectral distortion upon increasing the analyte concentration, but an overall reduction of the fluorescence intensity. This result indicates that there is a single fluorescent species in solution, namely the EtQxBox, and that the analytes act as quencher.

A variety of molecular interactions can result in quenching processes, including molecular rearrangements, energy transfer, complex formation, and collisional quenching. Although the effect is more pronounced for benzonitrile, one cannot readily conclude that fluorescence quenching is related to the receptor-analyte complexation only, since also diffusive collision could play a role with the same quencher. When both
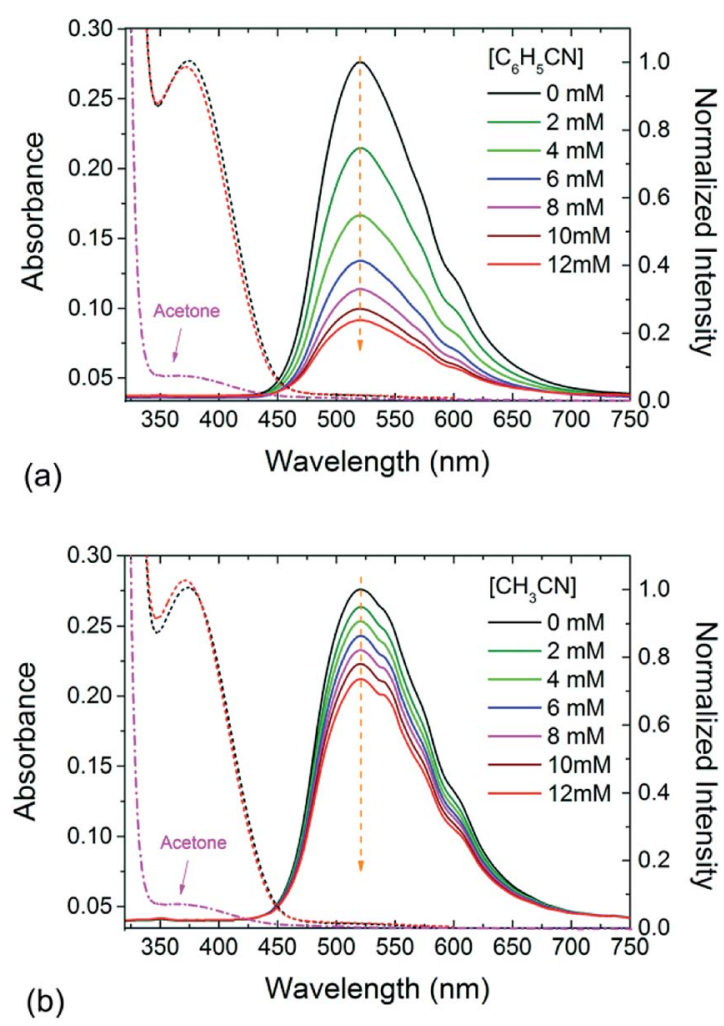

Fig. 1 Fluorescence intensity of EtQxBox $(0.5 \mathrm{mM})$ in acetone (black line) and at increasing concentrations (2-12 $\mathrm{mM}$ ) of (a) benzonitrile $\left(\mathrm{C}_{6} \mathrm{H}_{5} \mathrm{CN}\right)$ and (b) acetonitrile $\left(\mathrm{CH}_{3} \mathrm{CN}\right)$. Absorption spectra of the EtQxBox $(0.5 \mathrm{mM})$ solution and after addition of the quencher $(12 \mathrm{mM})$ are reported as black and red dashed curves, respectively. 


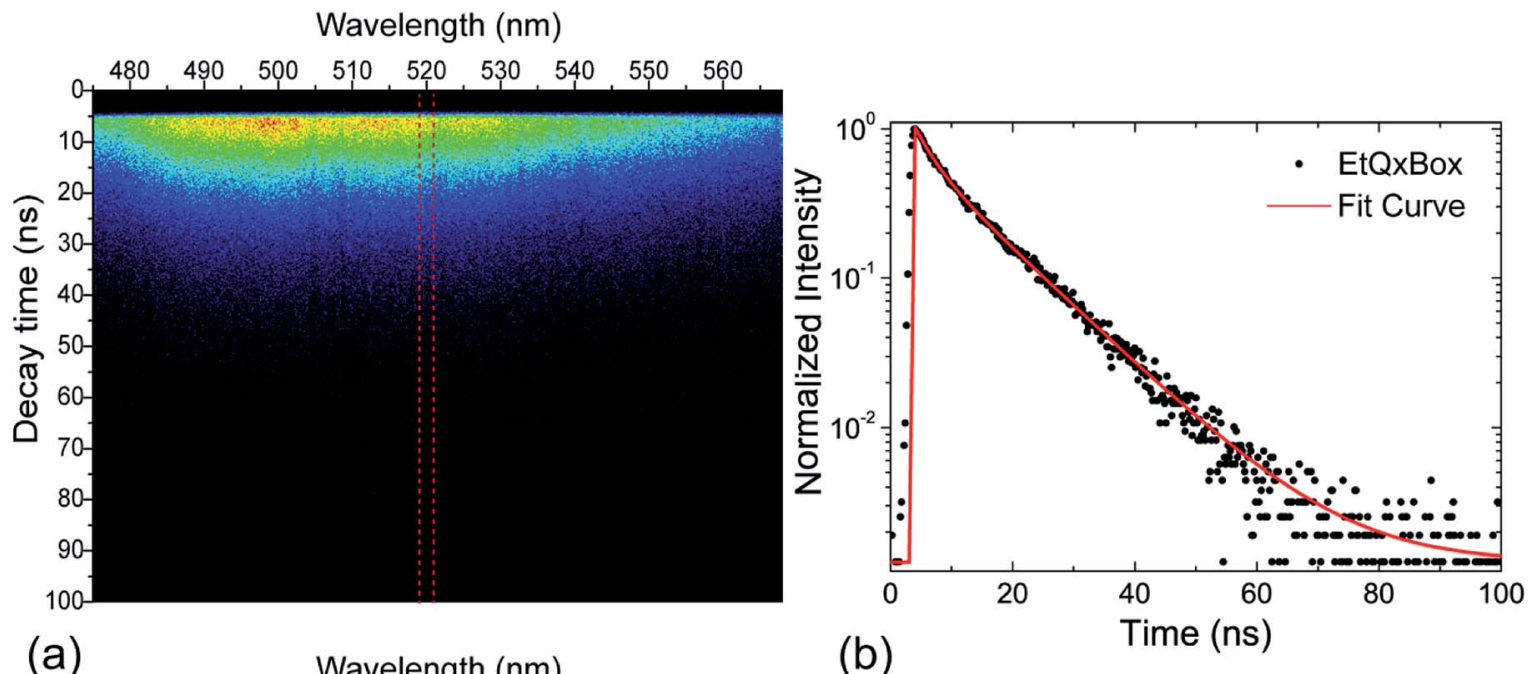

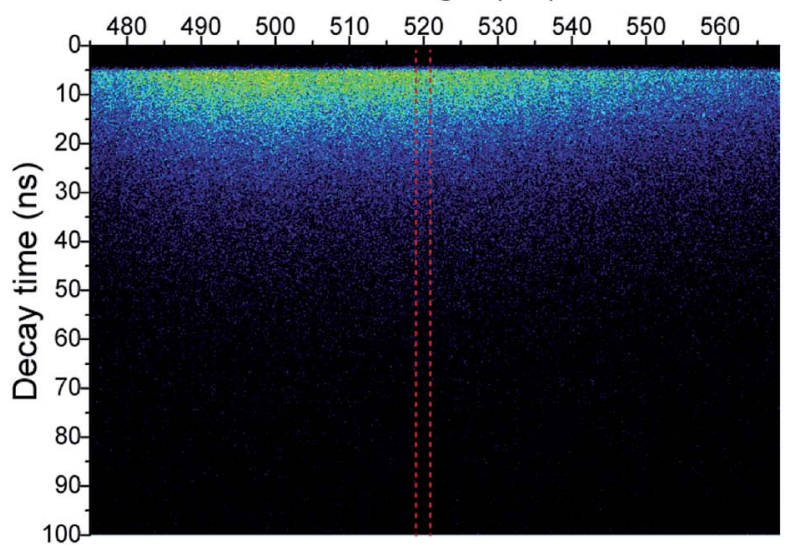

(c)

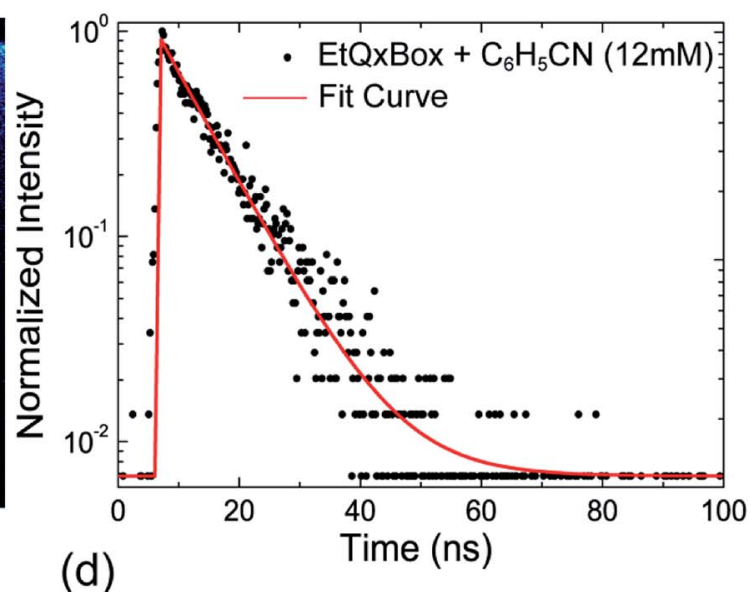

Fig. 2 Streak camera image for (a) EtQxBox $(0.5 \mathrm{mM})$ solution and (c) after addition of benzonitrile (12 mM). Fluorescence intensity (black circles) at $520 \mathrm{~nm}$ for (b) the EtQxBox $(0.5 \mathrm{mM})$ solution and (d) the EtQxBox-benzonitrile $(0.5-12 \mathrm{mM})$ solution as a function of time.

dynamic and static quenching occur, a modified form of the Stern-Volmer equation is typically used: ${ }^{9}$

$$
\frac{I_{0}}{I}=\left(1+K_{\mathrm{A}}[\mathrm{Q}]\right)\left(1+K_{\mathrm{D}}[\mathrm{Q}]\right)
$$

where $I_{0}$ and $I$ are the fluorescence intensities of the sole fluorophore in solution and after the addition of the quencher, [Q] is the concentration of quencher, $K_{\mathrm{A}}$ and $K_{\mathrm{D}}$ are the association rate and the dynamic quenching constants, respectively. The first term on the right side of eqn (1) accounts for the formation of non-fluorescent fluorophore-quencher complexes; the second is related to a non-radiative energy transfer due to the collisions between emitter and quencher. The dynamic quenching constant is given by $K_{\mathrm{D}}=k_{\mathrm{q}} \tau_{0}$, where $k_{\mathrm{q}}$ is the bimolecular quenching constant and $\tau_{0}$ the lifetime of the fluorophore in the absence of the quencher. ${ }^{9}$ Although a quadratic dependence of $I_{0} / I$ on the quencher concentration is expected for combined static and dynamic quenching, steady-state fluorescence spectroscopy does not allow to distinguish the static and dynamic rate constants. In this regard, fluorescence lifetimes measurement provides a direct method to evaluate the dynamic constant $K_{\mathrm{D}}$. By removing the non-fluorescent complexed fluorophores from observation, static quenching does not affect lifetime of the uncomplexed fluorophores. On the other hand, collisional quenching is a rate process that depopulates the fluorophore excited state, therefore

$$
\frac{\tau_{0}}{\tau}=\left(1+K_{\mathrm{D}}[\mathrm{Q}]\right)
$$

where $\tau$ is the lifetime of the fluorophore at the quencher concentration $[\mathrm{Q}]$.

Combining eqn (1) and (2), the association constant $K_{\mathrm{A}}$ can be evaluated from

$$
\left(\frac{I_{0}}{I}\right) /\left(\frac{\tau_{0}}{\tau}\right)=\left(1+K_{\mathrm{A}}[\mathrm{Q}]\right)
$$

Fig. 2 shows the streak camera images for (a) EtQxBox $0.5 \mathrm{mM}$ solution and (c) the EtQxBox-benzonitrile solution (0.5$12 \mathrm{mM}$ ), both in acetone. The images have been acquired in photon counting mode (exposure time $=35.21 \mathrm{~ms}$, \# of exposures $=10^{4}$ ). The red dashed lines define the $5 \mathrm{~nm}$ wide spectral 
Table 1 Lifetime and intensity ratios deduced from static and timeresolved fluorescence measurements of $0.5 \mathrm{mM}$ acetone solution of EtQxBox at different concentrations of benzonitrile guest ( $Q$ )

\begin{tabular}{llrll}
\hline$Q[\mathrm{mM}]$ & $I_{0} / I$ & $\tau(\mathrm{ns})$ & $\tau_{0} / \tau$ & $\left(I_{0} / I\right) /\left(\tau_{0} / \tau\right)$ \\
\hline 0 & 1.0 & 10.20 & 1.0 & 1.0 \\
2 & 1.33 & 9.99 & 1.02 & 1.30 \\
4 & 1.82 & 9.54 & 1.07 & 1.70 \\
6 & 2.39 & 9.16 & 1.11 & 2.15 \\
8 & 2.99 & 8.86 & 1.15 & 2.60 \\
10 & 3.73 & 8.31 & 1.23 & 3.03 \\
12 & 4.23 & 8.03 & 1.27 & 3.33 \\
\hline
\end{tabular}

region of interest (ROI) centered at the maximum intensity (520 $\mathrm{nm}$ ) used to evaluate the fluorescence lifetime from the average emission vs. time. Black dots in Fig. $2 \mathrm{~b}$ and $\mathrm{d}$ represent the Time-Correlated Single Photon Counting (TCSPC) data obtained in the fluorescence lifetime measurements related to the EtQxBox $(0.5 \mathrm{mM})$ and EtQxBox-benzonitrile (0.5-12 mM) solutions, respectively. The fluorescence intensity decay is deconvoluted with a multi-exponential function (solid lines in Fig. $2 \mathrm{~b}$ and d) and the weighted average lifetimes $\tau$ are reported in Tables 1 and 2 versus the $\mathrm{C}_{6} \mathrm{H}_{5} \mathrm{CN}$ and $\mathrm{CH}_{3} \mathrm{CN}$ concentration respectively, together with the steady-state fluorescence intensity ratios evaluated at $520 \mathrm{~nm}$ (Fig. 1). The two analytes yield a similar luminescence decay of the EtQxBox, whose lifetime $(10.20 \mathrm{~ns})$ is shortened to $8.03 \mathrm{~ns}$ for benzonitrile and to $8.26 \mathrm{~ns}$ for acetonitrile, at $12 \mathrm{mM}$. Conversely, the steady-state fluorescence quenching $I_{0} / I$ induced by benzonitrile is much larger than of acetonitrile.

In Fig. 3, the steady-state intensity $I_{0} / I$ (red) and lifetime $\tau_{0} / \tau$ (black) quenching data are represented in Stern-Volmer plots. The positive deviation of the $I_{0} / I$ data from the $\tau_{0} / \tau$ is evident in Fig. 3a, whose quadratic upward-curving behavior typical of combined dynamic and static quenching (eqn (1)) is confirmed by best curve fitting (red solid line). From the slope of the $\tau_{0} / \tau$ data and eqn (2) we estimate equal dynamic constant values for benzonitrile and acetonitrile $K_{\mathrm{D}}=(21 \pm 1) \mathrm{M}^{-1}$ and, considering the measured fluorescence lifetime $\tau_{0}=10.2 \mathrm{~ns}$, the bimolecular quenching constant $k_{\mathrm{q}} \equiv K_{\mathrm{D}} / \tau_{0}=(2.0 \pm 0.1) \times 10^{9}$ $\mathrm{M}^{-1} \mathrm{~s}^{-1}$.

On the contrary, the association constants, evaluated from the best fits of the $\left(I_{0} \tau / I \tau_{0}\right)$ data and eqn (3), are very different for

Table 2 Lifetime and intensity ratios deduced from static and timeresolved fluorescence measurements of $0.5 \mathrm{mM}$ acetone solution of EtQxBox at different concentrations of acetonitrile guest $(Q)$

\begin{tabular}{llrll}
\hline$Q[\mathrm{mM}]$ & $I_{0} / I$ & $\tau(\mathrm{ns})$ & $\tau_{0} / \tau$ & $\left(I_{0} / I\right) /\left(\tau_{0} / \tau\right)$ \\
\hline 0 & 1.0 & 10.20 & 1.0 & 1.0 \\
2 & 1.06 & 9.94 & 1.02 & 1.03 \\
4 & 1.10 & 9.50 & 1.07 & 1.03 \\
6 & 1.16 & 9.03 & 1.13 & 1.02 \\
8 & 1.23 & 8.69 & 1.17 & 1.05 \\
10 & 1.27 & 8.42 & 1.21 & 1.05 \\
12 & 1.36 & 8.26 & 1.23 & 1.10
\end{tabular}

benzonitrile $K_{\mathrm{A}}^{\mathrm{b}}=(203 \pm 6) \mathrm{M}^{-1}$ and acetonitrile $K_{\mathrm{A}}^{\mathrm{a}}=(6 \pm 1)$ $\mathrm{M}^{-1}$.

These results prove that: (i) collisional quenching is aspecific, its contribution to the fluorescence quenching being similar for benzonitrile and acetonitrile; (ii) the pure static quenching due to non-fluorescent complex formation is strongly selective toward the aromatic analyte, whose association constant is more than 30 times larger than the aliphatic one. Moreover, while the moderate fluorescence reduction induced by acetonitrile (Fig. 1b) is mostly due to collisional encounters with the receptor $\left(K_{\mathrm{A}}^{\mathrm{a}}<K_{\mathrm{D}}\right)$, the receptor-analyte complexation process dominates the fluorescence quenching mechanism in the case of benzonitrile $\left(K_{\mathrm{A}}^{\mathrm{b}} \gg K_{\mathrm{D}}\right)$.

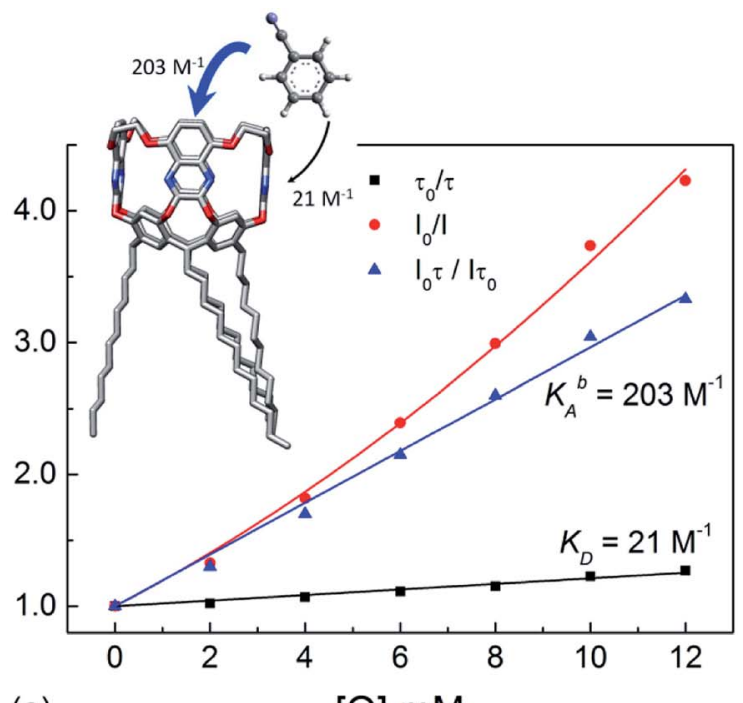

(a)

[Q] $\mathrm{mM}$

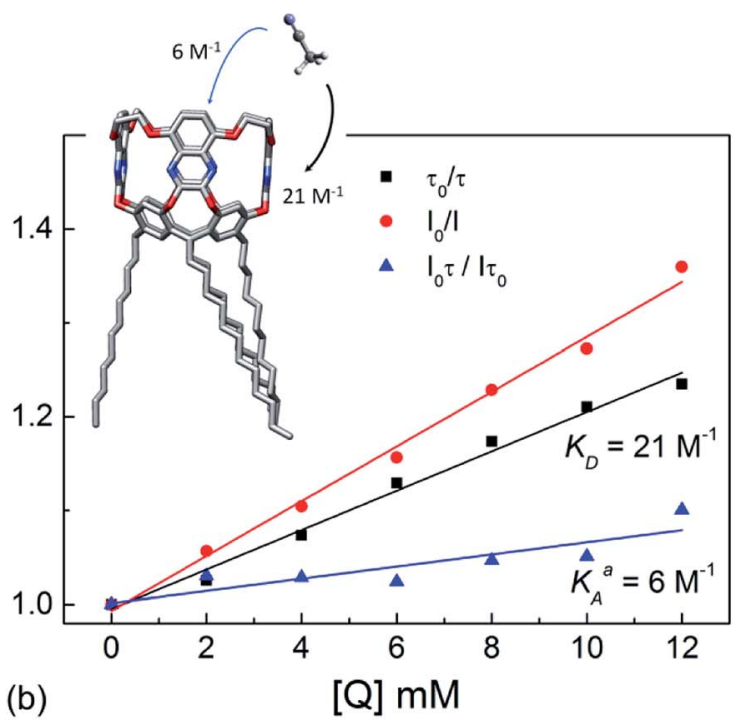

Fig. 3 Stern-Vomer quenching analysis of EtQxBox $(0.5 \mathrm{mM}$ in acetone) with (a) benzonitrile and (b) acetonitrile. The steady-state intensity $I_{0} / /$ (red dots) and lifetime $\tau_{0} / \tau$ (black squares) quenching data are reported versus the analyte concentration, together with the static quenching contribution $\left(I_{0} \tau / / \tau_{0}\right)$ (blue triangles). The red, black and blue curves are best fits of experimental data by eqn (1)-(3), respectively. Cavitand structure is MM3 minimized model. 
Nonetheless, the occurrence of aspecific dynamic quenching makes the sole steady-state fluorescence results inappropriate to detect aromatic analytes faithfully in presence of aliphatic interferents, exposing to false positive response. Reliable sensing and quantification of the target analyte concentration based on fluorescence spectroscopy is possible combining static and time-resolved measurements.

\section{Conclusions}

The specificity of a chemical detection approach depends not only on the selectivity of the receptor, but also of the transduction scheme chosen for translating the molecular recognition event into a physical signal. Herein, a combined steadystate and time-resolved fluorescence investigation has been carried out aimed at assessing the complexation capability of EtQxBox in solution toward aromatic and aliphatic VOCs. The quenching behavior of the fluorescent receptor exhibits different analyte concentration dependence for intensity and lifetime measurements, the positive deviation of the luminescence intensity versus lifetime being the first evidence of the predominant ground-state complex formation for the aromatic analyte. Quantitative estimation of the dynamic and association constants for benzonitrile and acetonitrile confirms the high selectivity of EtQxBox toward aromatics. Nonetheless, aspecific collisional quenching coexists, making steady-state-only fluorescence transduction mode prone to false positives in presence of aliphatic interferents.

\section{Conflicts of interest}

There are no conflicts to declare.

\section{Acknowledgements}

A. A., G. P., A. D. L. and P. P. acknowledge the Beyond-Nano Facility of CNR-Nanotec (Rende) for the use of time-resolved fluorescence spectrograph. R. P. and E. D acknowledge the Centro Interfacoltá di Misure "G. Casnati" for the use of NMR and MALDI MS facilities.

\section{References}

1 V. Gubala, L. F. Harris, A. J. Ricco, M. X. Tan and D. E. Williams, Anal. Chem., 2012, 84, 487-515.

2 H. Hierlemann and R. Gutierrez-Osuna, Chem. Rev., 2008, 108, 563-613.

3 The lock-and-key principle: the state of the art 100 years on, J. P. Behr, John Wiley \& Sons, Chichester, 1995.

4 J. W. Steed, J. L. Atwood and P. A. Gale, Definition and emergence of supramolecular chemistry, John Wiley \& Sons, Chichester, 2012.

5 G. R. Desiraju, Nature, 2001, 412, 397-400.

6 J. J. Lavigne and E. V. Anslyn, Angew. Chem., Int. Ed., 2001, 40, 3118-3130.

7 M. Melegari, C. Massera, R. Pinalli, R. M. Yebeutchou and E. Dalcanale, Sens. Actuators, B, 2013, 179, 74-80.

8 A. Aprile, F. Ciuchi, R. Pinalli, E. Dalcanale and P. Pagliusi, J. Phys. Chem. Lett., 2016, 7, 3022-3026.

9 J. R. Lakowicz, Principles of Fluorescence Spectroscopy, Springer, Singapore, 2006.

10 L. Prodi, New J. Chem., 2005, 29, 20-31.

11 A. P. De Silva, H. N. Gunaratne, T. Gunnlaugsson, A. J. Huxley, C. P. McCoy, J. T. Rademacher and T. E. Rice, Chem. Rev., 1997, 97, 1515-1566.

12 E. Biavardi, G. Battistini, M. Montalti, R. M. Yebeutchou, L. Prodi and E. Dalcanale, ChemComm, 2008, 1638-1640.

13 J. S. Kim and D. T. Quang, Chem. Rev., 2007, 107, 3780-3799. 14 D. Masseroni, E. Biavardi, D. Genovese, E. Rampazzo, L. Prodi and E. Dalcanale, ChemComm, 2015, 51, 1279912802.

15 J. W. Trzcinski, R. Pinalli, N. Riboni, A. Pedrini, F. Bianchi, S. Zampolli, I. Elmi, C. Massera, F. Ugozzoli and E. Dalcanale, ACS Sens., 2017, 2, 590-598.

16 N. Riboni, J. W. Trzcinski, F. Bianchi, C. Massera, R. Pinalli, L. Sidisky, E. Dalcanale and M. Careri, Anal. Chim. Acta, 2016, 905, 79-84.

17 R. Pinalli, A. Pedrini and E. Dalcanale, Chem.-Eur. J., 2018, 24, 1010-1019. 